\title{
Acceptance of Disability, Coping Style, Perceived Social Support and Quality of Life Among Patients With Chronic Lymphedema: a Cross-sectional Study
}

\section{Zehao Huang ( $\nabla$ huangzh@link.cuhk.edu.hk)}

The Chinese University of Hong Kong The Nethersole School of Nursing https://orcid.org/0000-0001-5518-858X

\section{Siyu Wu}

Foshan Hospital of Traditional Chinese Medicine

\section{Research Article}

Keywords: Quality of life, Acceptance of disability, Coping styles, Perceived social support, Chronic lymphedema, Chinese

Posted Date: November 29th, 2021

DOl: https://doi.org/10.21203/rs.3.rs-1002210/v1

License: (9) (i) This work is licensed under a Creative Commons Attribution 4.0 International License. Read Full License

Version of Record: A version of this preprint was published at Supportive Care in Cancer on January 24th, 2022. See the published version at https://doi.org/10.1007/s00520-022-06855-4. 


\section{Abstract}

Objective: To examine acceptance of disability, coping style, perceived social support, and quality of life and to explore the relationships between acceptance of disability, coping style, perceived social support and quality of life among Chinese patients with chronic lymphedema.

Methods: Chronic lymphedema patients were recruited from five tertiary hospitals between May and July 2020 in China. Recruited patients were assessed for quality of life (QOL), acceptance of disability (AOD), coping styles, perceived social support (PSS), and sociodemographic and disease-related factors. Multivariate linear regression models were conducted to examine the multivariate effect of AOD, coping style, PSS, and sociodemographic and disease-related factors on QOL.

Results: A total of 163 chronic lymphedema patients were recruited. The mean score of QOL was 2.23 (SD=0.68). AOD, number of symptoms, acceptance-resignation, avoidance, degree of pain, PSS, and educational level were found to be significant predictors of QOL.

Conclusion: Chinese patients with chronic lymphedema had moderate levels of QOL. The QOL and specific domains of patients were affected by different factors. Special attention and targeted interventions should be given to improve patients' QOL.

\section{Introduction}

Chronic lymphedema is traditionally viewed as an incurable and progressive condition, characterized by progressive swelling of one or more regions of the body that has been present for more than 3 months [1]. It occurs when there is a dysfunction of the lymph drainage system, either congenital or acquired, resulting in the accumulation of proteinrich fluid in the interstitial spaces [2]. It is estimated that approximately 140 250 million individuals worldwide suffer from lymphedema [3]. A recent study showed that in Germany, the total costs of lymphedema treatment and management per patient per year were $€ 5784$ [4]. Moreover, chronic lymphedema, if not properly treated and managed, often adversely affects the limbs of patients and is associated with functional deficits and even disability, severe symptoms, and various complications, which brings challenges to patients' psychological and emotional wellbeing and social adaptation and impairs the QOL $[5,6]$.

Effective psychosocial interventions are required to help chronic lymphedema patients cope with their functional deficits, activity limitations, and participation restriction and accept their disabilities [7]. Acceptance of disability, a significant element in psychosocial adaptation, refers to through shifts of value system, an individual perceives that the losses caused by disability do not impair the existing normal abilities and meaning of existence [8]. Former studies showed a significant correlation between acceptance of disability and quality of life among patients with different chronic diseases $[9,10]$.

Coping is generally defined as the person's cognitive and behavioral efforts to manage external/internal stressors that are appraised as taxing or exceeding one's resources or to alleviate the negative emotions and conflict caused by stress [11]. Individuals may adopt divergent coping strategies when facing stressful situations, and different coping styles are closely related to the person's psychosocial adjustment and quality of life [12]. Previous studies illustrated that patients who prefer adaptive or active coping styles had a better quality of life, while maladaptive or avoidancetype coping can worsen their quality of life $[13,14]$. 
Perceived social support, people's perceptions about how much support from the social network would be accessible and the quality of the support during stressful periods of life, is regarded as a protective factor for a person's psychological, cognitive, and behavioral adaptation when under stress; in contrast, received support is the actual support the individual receives $[15,16]$. A large body of evidence showed that perceived social support has a positive effect on minimizing patients' physical disability and promoting their psychosocial adjustment, thereby improving their quality of life $[17,18]$.

Therefore, based on the theory of Psychosocial Adaptation to Chronic Illness and Disability (CID) [19], we conducted this study to determine the correlation between acceptance of disability, coping style, perceived social support and quality of life among Chinese patients with chronic lymphedema and provide evidence for the development of psychosocial interventions that are focusing on improving chronic lymphedema patients' QoL.

\section{Methods}

\subsection{Study design and participants}

A cross-sectional design was conducted to collect data. We recruited consecutive chronic lymphedema patients from five tertiary hospitals in southern China between May and July 2020. The inclusion criteria were as follows: (1) clinically diagnosed with chronic lymphedema; (2) lymphedema occurs in limbs and presents for more than 3 months; (3) age of 18 years or above; (4) able to understand and complete questionnaires; (5) agree to participate. Patients with cognitive impairment, mental illness, or severe physical illnesses were excluded.

\subsection{Measures}

We used a self-designed questionnaire to obtain sociodemographic and disease-related information. The sociodemographic information included gender, age, marital status, educational level, monthly income, place of residence, and employment status. The disease-related information included the type of lymphedema, localization of lymphedema, lymphedema stages, comorbidities, time since diagnosis, number of symptoms, degree of pain, and sexual distress caused by lymphedema.

The acceptance of disability of patients with chronic lymphedema was assessed using the revised version of the Acceptance of Disability Scale (ADS-R) [20]. The revised scale contains 32 items and is divided into 4 dimensions: enlargement of scope of values, subordination of physique, containment of disability effect, and transformation from comparative values to asset values. Each item is scored on a 4-point Likert scale, ranging from 1= 'strongly disagree' to $4=$ 'strongly agree'. The total score of the scale ranges from 32 to 128 , where a higher score represents a better level of disability acceptance. A total score of $32-64,65$ to 96 , and 97 to 128 indicate a low, moderate, and high level of disability acceptance, respectively. The Cronbach's a coefficient in our study was 0.946 .

The Medical Coping Modes Questionnaire (MCMQ) was used to assess the coping strategies that participants may adopt when facing medical events [21]. It contains 20 items (one item was added with consideration of maintaining the original meaning of the questionnaire) and is divided into 3 dimensions, including confrontation, avoidance, and acceptance-resignation. Each item is scored on a 4-point Likert scale, ranging from 1 to 4 . A higher score on each dimension indicates greater likelihoods that the participants will adopt the corresponding coping style. The Cronbach's a coefficient in our study was 0.725 .

We assessed the perceived social support of patients with chronic lymphedema using the Multidimensional Scale of Perceived Social Support (MSPSS) [22]. The MSPSS consists of 12 items that measure perceived social support in 
three dimensions: friend support, family support, and significant other support. Each item is scored on a 7-point Likert scale, ranging from $1=$ 'very strongly disagree' to 4 = 'very strongly agree'. The total score of the scale ranges from 12 to 84 , where a higher score represents greater social support perceived by an individual. A total score of $12-35,36$ to 61 , and 62 to 84 indicate a low, moderate, and high level of disability acceptance, respectively. The Cronbach's a coefficient in our study was 0.964 .

The short version of the Quality of Life Questionnaire for Persons Living with Lymph Conditions (FLQA-LS) was adopted to evaluate the quality of life in patients with chronic lymphedema [23]. The FLQA-LS contains 33 items, of which 1 30 items are divided into five subscales, namely, physical impairments, daily life, social life, mental condition, and Therapy of lymphatic disorder. Each item is scored on a 5-point Likert scale, ranging from 1= 'never/not at all' to 5 = 'always/very'; The higher the score, the lower the quality of life. In addition, items 31 33 are applied to assess the generic QOL of patients in the preceding week, using the numeric scale ranging from 0 (very poor) to 10 (very good). After getting permission from the original authors of the scale, we created the Chinese version using translation, back-translation, and cultural adaptation. The content validity index (CVI) of the Chinese version scale was evaluated by 6 experts. The item-content validity index (I-CVI) was 0.67 1, and item 2 was deleted because it was less than 0.78; The scale level CVI/universal agreement (S-CVI/UA) was 0.97, and the S-CVI/Ave was 0.99. The Cronbach's a coefficient in our study was 0.828 .

\subsection{Statistical analysis}

The analyses were carried out using the SPSS version 25.0 for Windows. A two-sided $p<0.05$ was considered statistically significant. Descriptive statistical analyses were conducted using means and standard deviations (SDs) to present continuous variables and frequencies to show categorical variables. For each sociodemographic and disease-related variable, an independent sample t-test or one-way analysis of variance (ANOVA) was performed to compare their mean scores on the FLQA-LS. Pearson correlation analysis was used to explore the relationships among acceptance of disability, coping style, perceived social support, and quality of life. Multiple linear regression models were performed to assess the relative importance of these sociodemographic and disease-related variables, acceptance of disability, coping style, and perceived social support in explaining the quality of life.

\section{Results}

\subsection{Characteristics of participants}

A total of 163 patients were finally enrolled in the study with a response rate of $94.8 \%$. The sample included 160 females (98.2\%) and 3 males (1.8\%), with ages ranging from 32 75 years. The detailed sociodemographic and disease-related characteristics of patients with chronic lymphedema are presented in Table 1. 
Table 1

Characteristics of Chinese patients with chronic lymphedema $(\mathrm{N}=163)$

\begin{tabular}{|c|c|}
\hline Variables & $\mathbf{N}(\%)$ \\
\hline \multicolumn{2}{|l|}{ Gender } \\
\hline Male & $3(1.8)$ \\
\hline Female & $160(98.2)$ \\
\hline \multicolumn{2}{|l|}{ Age (Years) } \\
\hline $32 \sim 44$ & $31(19.0)$ \\
\hline $45 \sim 59$ & $102(62.6)$ \\
\hline $60 \sim 75$ & $30(18.4)$ \\
\hline \multicolumn{2}{|l|}{ Marital status } \\
\hline Married & $147(90.2)$ \\
\hline Single/divorced/widowed/separated & $16(9.8)$ \\
\hline \multicolumn{2}{|l|}{ Educational level } \\
\hline Below college & $88(54.0)$ \\
\hline College or above & $75(46.0)$ \\
\hline \multicolumn{2}{|l|}{ Monthly income (CNY) } \\
\hline$\leq 2000$ & $25(15.3)$ \\
\hline $2001 \sim 4000$ & $44(27.0)$ \\
\hline $4001 \sim 6000$ & $36(22.1)$ \\
\hline $6001 \sim 8000$ & $26(16.0)$ \\
\hline $8001 \sim 10000$ & $13(8.0)$ \\
\hline$>10000$ & $19(11.7)$ \\
\hline \multicolumn{2}{|l|}{ Place of residence } \\
\hline Urban & $137(84.0)$ \\
\hline Rural & $26(16.0)$ \\
\hline \multicolumn{2}{|l|}{ Employment status } \\
\hline Full-time job & $58(35.6)$ \\
\hline Unemployed & $105(64.4)$ \\
\hline Type of lymphedema & \\
\hline
\end{tabular}

Symptoms: pain, fatigue, heaviness, swelling, tightness, tingling, discomfort, weakness, numbness.

Comorbidities: lymphangitis, Lymphatic cyst, lymphatic leakage, cellulitis, diabetes mellitus, infection, leg ulcer, diabetic foot ulcer, elephantiasis, lymphangiosarcoma, hypertension, obesity, heart failure. 


\begin{tabular}{|c|c|}
\hline Variables & $N(\%)$ \\
\hline Primary & $7(4.3)$ \\
\hline Secondary & $156(95.7)$ \\
\hline \multicolumn{2}{|c|}{ Localization of lymphedema } \\
\hline Arm & $97(59.5)$ \\
\hline Leg & $55(33.7)$ \\
\hline Both & $11(6.8)$ \\
\hline \multicolumn{2}{|c|}{ Lymphedema stages } \\
\hline Stage $0 \sim 1$ & $104(63.8)$ \\
\hline Stage 2 3 & $59(36.2)$ \\
\hline \multicolumn{2}{|c|}{ Number of comorbidities* } \\
\hline 0 & $98(60.1)$ \\
\hline 1 & $42(25.8)$ \\
\hline 2 & $16(9.8)$ \\
\hline$\geq 3$ & $7(4.3)$ \\
\hline \multicolumn{2}{|c|}{ Time since diagnosis } \\
\hline $\mathrm{a}<6$ (months) & 45 (27.6) \\
\hline $6 \leq a<12$ (months) & $13(8.0)$ \\
\hline $1 \leq \mathrm{a}<2$ (years) & $30(18.4)$ \\
\hline $2 \leq a<5$ (years) & $53(32.5)$ \\
\hline $5 \leq a<10$ (years) & $14(8.6)$ \\
\hline$a \geq 10$ (years) & $8(4.9)$ \\
\hline \multicolumn{2}{|c|}{ Number of symptoms* } \\
\hline 0 & $9(5.5)$ \\
\hline 1 & $40(24.5)$ \\
\hline 2 & $35(21.5)$ \\
\hline$\geq 3$ & $79(48.5)$ \\
\hline \multicolumn{2}{|l|}{ Degree of pain } \\
\hline No pain & $100(61.3)$ \\
\hline Mild pain & $41(25.2)$ \\
\hline
\end{tabular}

Symptoms: pain, fatigue, heaviness, swelling, tightness, tingling, discomfort, weakness, numbness.

Comorbidities: lymphangitis, Lymphatic cyst, lymphatic leakage, cellulitis, diabetes mellitus, infection, leg ulcer, diabetic foot ulcer, elephantiasis, lymphangiosarcoma, hypertension, obesity, heart failure. 


\begin{tabular}{|lc|}
\hline Variables & $\mathbf{N}(\%)$ \\
\hline Moderate pain & $22(13.5)$ \\
\hline Sexual distress caused by lymphedema & $28(17.2)$ \\
\hline Yes & $135(82.8)$ \\
\hline None & \\
\hline Symptoms: pain, fatigue, heaviness, swelling, tightness, tingling, discomfort, weakness, numbness. \\
\hline $\begin{array}{l}\text { Comorbidities: lymphangitis, Lymphatic cyst, lymphatic leakage, cellulitis, diabetes mellitus, infection, leg ulcer, } \\
\text { diabetic foot ulcer, elephantiasis, lymphangiosarcoma, hypertension, obesity, heart failure. }\end{array}$ \\
\hline
\end{tabular}

\subsection{Acceptance of disability, coping style, perceived social support, and quality of life}

As demonstrated in Table 2, the mean total score of AOD was 83.69 (SD=15.17), indicating that patients had moderate levels of AOD. Concerning coping styles, the confrontation domain obtained the highest score (2.32 \pm 0.44$)$, indicating that patients tended to adopt this kind of coping strategy. Moreover, the mean total score of PSS was 57.71 $(S D=11.24)$, indicating that patients had moderate levels of PSS. Furthermore, the mean total score of QOL was 2.23 $(S D=0.68)$ and standardized scores (standardized scores=mean/total score*100\%) was $44.6 \%$, indicating that patients' mean score is slightly lower than $50 \%$ of the possible total score, which implies moderate levels of QOL. 
Table 2

Acceptance of disability, coping style, and perceived social support, and quality of life in patients with chronic lymphedema $(\mathrm{N}=163)$

\begin{tabular}{|c|c|c|}
\hline Subscale & Scale/Domain level scores mean (SD) & Item level scores mean (SD) \\
\hline \multicolumn{3}{|l|}{ ADS-R } \\
\hline Total acceptance & $83.69 \pm 15.17$ & $2.62 \pm 0.47$ \\
\hline Enlargement & $26.27 \pm 5.06$ & $2.92 \pm 0.56$ \\
\hline Subordination & $11.37 \pm 3.16$ & $2.27 \pm 0.63$ \\
\hline Containment & $21.75 \pm 6.71$ & $2.42 \pm 0.75$ \\
\hline Transformation & $24.29 \pm 5.14$ & $2.70 \pm 0.57$ \\
\hline \multicolumn{3}{|l|}{ MCMQ } \\
\hline Confrontation & $18.51 \pm 3.52$ & $2.32 \pm 0.44$ \\
\hline Avoidance & $14.72 \pm 2.59$ & $2.10 \pm 0.38$ \\
\hline Acceptance-resignation & $10.01 \pm 3.28$ & $1.99 \pm 0.66$ \\
\hline \multicolumn{3}{|l|}{ MSPSS } \\
\hline Total perceived social support & $57.71 \pm 11.24$ & $4.76 \pm 0.94$ \\
\hline Family support & $22.58 \pm 4.08$ & $5.64 \pm 1.02$ \\
\hline Friend support & $17.69 \pm 5.66$ & $4.42 \pm 1.42$ \\
\hline Significant other support & $16.90 \pm 4.68$ & $4.23 \pm 1.17$ \\
\hline \multicolumn{3}{|l|}{ FLQA-LS } \\
\hline Total QOL & $64.57 \pm 19.75$ & $2.23 \pm 0.68$ \\
\hline Physical impairments & $11.57 \pm 4.30$ & $2.31 \pm 0.86$ \\
\hline Daily life & $13.36 \pm 4.84$ & $2.23 \pm 0.81$ \\
\hline Social life & $11.19 \pm 5.06$ & $1.87 \pm 0.84$ \\
\hline Mental condition & $15.47 \pm 4.75$ & $2.58 \pm 0.79$ \\
\hline Therapy of lymphatic disorder & $12.97 \pm 5.36$ & $2.16 \pm 0.89$ \\
\hline
\end{tabular}

\subsection{Sociodemographic and disease-related characteristics associated with quality of life}

The sociodemographic and disease-related characteristics associated with quality of life and its five domains are shown in Table 3. As shown, the total QOL was significantly associated with the educational level, the place of residence, the number of symptoms, the degree of pain, and the sexual distress caused by lymphedema. Among all the five domains, physical impairments were significantly related to the number of comorbidities, the number of symptoms, the degree of pain, and the sexual distress caused by lymphedema. The correlative factors of daily life included the educational level, the lymphedema stage, the number of symptoms, the degree of pain, and the sexual 
distress caused by lymphedema. Significant associations were observed between social life and the educational level, the place of residence, the employment status, the localization of lymphedema, the lymphedema stage, the number of symptoms, the degree of pain, and the sexual distress caused by lymphedema. Moreover, the associated factors of the mental condition were the educational level, the place of residence, the number of symptoms, the degree of pain, and the sexual distress caused by lymphedema. In addition, therapy of lymphatic disorder was significantly correlated to the educational level, the place of residence, the number of symptoms, and the degree of pain. 
Table 3

Sociodemographic and disease-related characteristics of quality of life $(\mathrm{N}=163)$

\begin{tabular}{|c|c|c|c|c|c|c|}
\hline Variables & $\begin{array}{l}\text { Total } \\
\text { QOL }\end{array}$ & $\begin{array}{l}\text { Physical } \\
\text { Impairments }\end{array}$ & $\begin{array}{l}\text { Daily } \\
\text { Life }\end{array}$ & $\begin{array}{l}\text { Social } \\
\text { Life }\end{array}$ & $\begin{array}{l}\text { Mental } \\
\text { Condition }\end{array}$ & $\begin{array}{l}\text { Therapy } \\
\text { of } \\
\text { Lymphatic } \\
\text { Disorder }\end{array}$ \\
\hline \multicolumn{7}{|l|}{ Gender } \\
\hline Male & $\begin{array}{l}2.50 \\
(0.38)\end{array}$ & $2.33(0.70)$ & $\begin{array}{l}2.22 \\
(0.38)\end{array}$ & $\begin{array}{l}2.11 \\
(0.42)\end{array}$ & $\begin{array}{l}3.00 \\
(0.33)\end{array}$ & $\begin{array}{l}2.83 \\
(0.73)\end{array}$ \\
\hline Female & $\begin{array}{l}2.22 \\
(0.69)\end{array}$ & $2.31(0.86)$ & $\begin{array}{l}2.23 \\
(0.81)\end{array}$ & $\begin{array}{l}1.86 \\
(0.85)\end{array}$ & $\begin{array}{l}2.57 \\
(0.80)\end{array}$ & $\begin{array}{l}2.15 \\
(0.89)\end{array}$ \\
\hline \multicolumn{7}{|l|}{ Age (Years) } \\
\hline $32 \sim 44$ & $\begin{array}{l}2.36 \\
(0.74)\end{array}$ & $2.31(0.86)$ & $\begin{array}{l}2.34 \\
(0.83)\end{array}$ & $\begin{array}{l}2.06 \\
(0.94)\end{array}$ & $\begin{array}{l}2.70 \\
(0.70)\end{array}$ & $\begin{array}{l}2.38 \\
(1.07)\end{array}$ \\
\hline $45 \sim 59$ & $\begin{array}{l}2.22 \\
(0.67)\end{array}$ & $2.36(0.85)$ & $\begin{array}{l}2.19 \\
(0.80)\end{array}$ & $\begin{array}{l}1.84 \\
(0.83)\end{array}$ & $\begin{array}{l}2.59 \\
(0.84)\end{array}$ & $\begin{array}{l}2.12 \\
(0.87)\end{array}$ \\
\hline $60 \sim 74$ & $\begin{array}{l}2.12 \\
(0.67)\end{array}$ & $2.15(0.91)$ & $\begin{array}{l}2.23 \\
(0.83)\end{array}$ & $\begin{array}{l}1.73 \\
(0.77)\end{array}$ & $\begin{array}{l}2.41 \\
(0.71)\end{array}$ & $\begin{array}{l}2.06 \\
(0.74)\end{array}$ \\
\hline \multicolumn{7}{|l|}{ Marital status } \\
\hline Married & $\begin{array}{l}2.23 \\
(0.69)\end{array}$ & $2.33(0.87)$ & $\begin{array}{l}2.21 \\
(0.81)\end{array}$ & $\begin{array}{l}1.87 \\
(0.87)\end{array}$ & $\begin{array}{l}2.58 \\
(0.79)\end{array}$ & $\begin{array}{l}2.18 \\
(0.90)\end{array}$ \\
\hline Single/divorced/widowed/separated & $\begin{array}{l}2.20 \\
(0.63)\end{array}$ & $2.21(0.80)$ & $\begin{array}{l}2.40 \\
(0.82)\end{array}$ & $\begin{array}{l}1.84 \\
(0.50)\end{array}$ & $\begin{array}{l}2.57 \\
(0.84)\end{array}$ & $\begin{array}{l}1.96 \\
(0.83)\end{array}$ \\
\hline \multicolumn{7}{|l|}{ Educational level } \\
\hline Below college & $\begin{array}{l}2.35 \\
(0.67)^{*}\end{array}$ & $2.41(0.88)$ & $\begin{array}{l}2.34 \\
(0.85)^{\star}\end{array}$ & $\begin{array}{l}2.02 \\
(0.84) \star \star \star\end{array}$ & $\begin{array}{l}2.70 \\
(0.77) *\end{array}$ & $\begin{array}{l}2.30 \\
(0.92) *\end{array}$ \\
\hline College or above & $\begin{array}{l}2.08 \\
(0.68)\end{array}$ & $2.21(0.82)$ & $\begin{array}{l}2.09 \\
(0.73)\end{array}$ & $\begin{array}{l}1.68 \\
(0.81)\end{array}$ & $\begin{array}{l}2.44 \\
(0.80)\end{array}$ & $\begin{array}{l}2.00 \\
(0.84)\end{array}$ \\
\hline \multicolumn{7}{|l|}{ Monthly income (CNY) } \\
\hline$\leq 2000$ & $\begin{array}{l}2.54 \\
(0.70)\end{array}$ & $2.47(0.71)$ & $\begin{array}{l}2.63 \\
(0.77)\end{array}$ & $\begin{array}{l}2.25 \\
(0.83)\end{array}$ & $\begin{array}{l}2.87 \\
(0.86)\end{array}$ & $\begin{array}{l}2.48 \\
(1.08)\end{array}$ \\
\hline $2001 \sim 4000$ & $\begin{array}{l}2.25 \\
(0.62)\end{array}$ & $2.38(0.84)$ & $\begin{array}{l}2.19 \\
(0.83)\end{array}$ & $\begin{array}{l}1.89 \\
(0.84)\end{array}$ & $\begin{array}{l}2.61 \\
(0.78)\end{array}$ & $\begin{array}{l}2.21 \\
(0.82)\end{array}$ \\
\hline $4001 \sim 6000$ & $\begin{array}{l}2.18 \\
(0.74)\end{array}$ & $2.23(1.06)$ & $\begin{array}{l}2.21 \\
(0.83)\end{array}$ & $\begin{array}{l}1.83 \\
(0.83)\end{array}$ & $\begin{array}{l}2.59 \\
(0.77)\end{array}$ & $\begin{array}{l}1.98 \\
(0.86)\end{array}$ \\
\hline $6001 \sim 8000$ & $\begin{array}{l}2.19 \\
(0.74)\end{array}$ & $2.33(0.89)$ & $\begin{array}{l}2.17 \\
(0.83)\end{array}$ & $\begin{array}{l}1.76 \\
(0.99)\end{array}$ & $\begin{array}{l}2.47 \\
(0.87)\end{array}$ & $\begin{array}{l}2.24 \\
(0.88)\end{array}$ \\
\hline $8001 \sim 10000$ & $\begin{array}{l}2.06 \\
(0.62)\end{array}$ & $1.97(0.59)$ & $\begin{array}{l}2.12 \\
(0.58)\end{array}$ & $\begin{array}{l}1.73 \\
(0.86)\end{array}$ & $\begin{array}{l}2.32 \\
(0.62)\end{array}$ & $\begin{array}{l}2.15 \\
(0.96)\end{array}$ \\
\hline$>10000$ & $\begin{array}{l}1.99 \\
(0.55)\end{array}$ & $2.14(0.79)$ & $\begin{array}{l}1.96 \\
(0.75)\end{array}$ & $\begin{array}{l}1.59 \\
(0.62)\end{array}$ & $\begin{array}{l}2.43 \\
(0.77)\end{array}$ & $\begin{array}{l}1.86 \\
(0.77)\end{array}$ \\
\hline
\end{tabular}




\begin{tabular}{|c|c|c|c|c|c|c|}
\hline Variables & $\begin{array}{l}\text { Total } \\
\text { QOL }\end{array}$ & $\begin{array}{l}\text { Physical } \\
\text { Impairments }\end{array}$ & $\begin{array}{l}\text { Daily } \\
\text { Life }\end{array}$ & $\begin{array}{l}\text { Social } \\
\text { Life }\end{array}$ & $\begin{array}{l}\text { Mental } \\
\text { Condition }\end{array}$ & $\begin{array}{l}\text { Therapy } \\
\text { of } \\
\text { Lymphatic } \\
\text { Disorder }\end{array}$ \\
\hline \multicolumn{7}{|c|}{ Place of residence } \\
\hline Urban & $\begin{array}{l}2.16 \\
(0.69) \star \star\end{array}$ & $2.27(0.87)$ & $\begin{array}{l}2.19 \\
(0.80)\end{array}$ & $\begin{array}{l}1.79 \\
(0.82) \star \star\end{array}$ & $\begin{array}{l}2.51 \\
(0.80) \star *\end{array}$ & $\begin{array}{l}2.06 \\
(0.83) \star \star\end{array}$ \\
\hline Rural & $\begin{array}{l}2.56 \\
(0.59)\end{array}$ & $2.52(0.81)$ & $\begin{array}{l}2.44 \\
(0.80)\end{array}$ & $\begin{array}{l}2.24 \\
(0.89)\end{array}$ & $\begin{array}{l}2.92 \\
(0.64)\end{array}$ & $\begin{array}{l}2.69 \\
(1.02)\end{array}$ \\
\hline \multicolumn{7}{|c|}{ Employment status } \\
\hline Full-time job & $\begin{array}{l}2.12 \\
(0.65)\end{array}$ & $2.23(0.87)$ & $\begin{array}{l}2.11 \\
(0.76)\end{array}$ & $\begin{array}{l}1.69 \\
(0.80) *\end{array}$ & $\begin{array}{l}2.49 \\
(0.80)\end{array}$ & $\begin{array}{l}2.09 \\
(0.84)\end{array}$ \\
\hline Unemployed & $\begin{array}{l}2.29 \\
(0.70)\end{array}$ & $2.36(0.86)$ & $\begin{array}{l}2.29 \\
(0.83)\end{array}$ & $\begin{array}{l}1.96 \\
(0.85)\end{array}$ & $\begin{array}{l}2.63 \\
(0.78)\end{array}$ & $\begin{array}{l}2.20 \\
(0.92)\end{array}$ \\
\hline \multicolumn{7}{|c|}{ Type of lymphedema } \\
\hline Primary & $\begin{array}{l}1.88 \\
(0.65)\end{array}$ & $2.11(0.84)$ & $\begin{array}{l}1.74 \\
(0.61)\end{array}$ & $\begin{array}{l}1.50 \\
(0.78)\end{array}$ & $\begin{array}{l}2.17 \\
(1.04)\end{array}$ & $\begin{array}{l}1.93 \\
(0.88)\end{array}$ \\
\hline Secondary & $\begin{array}{l}2.24 \\
(0.68)\end{array}$ & $2.32(0.86)$ & $\begin{array}{l}2.25 \\
(0.81)\end{array}$ & $\begin{array}{l}1.88 \\
(0.84)\end{array}$ & $\begin{array}{l}2.59 \\
(0.78)\end{array}$ & $\begin{array}{l}2.17 \\
(0.89)\end{array}$ \\
\hline \multicolumn{7}{|c|}{ Localization of lymphedema } \\
\hline Arm & $\begin{array}{l}2.15 \\
(0.67)\end{array}$ & $2.29(0.87)$ & $\begin{array}{l}2.15 \\
(0.80)\end{array}$ & $\begin{array}{l}1.71 \\
(0.77)^{\star}\end{array}$ & $\begin{array}{l}2.48 \\
(0.78)\end{array}$ & $\begin{array}{l}2.13 \\
(0.90)\end{array}$ \\
\hline Leg & $\begin{array}{l}2.30 \\
(0.71)\end{array}$ & $2.27(0.86)$ & $\begin{array}{l}2.26 \\
(0.78)\end{array}$ & $\begin{array}{l}2.05 \\
(0.93)\end{array}$ & $\begin{array}{l}2.68 \\
(0.84)\end{array}$ & $\begin{array}{l}2.22 \\
(0.89)\end{array}$ \\
\hline Both & $\begin{array}{l}2.54 \\
(0.53)\end{array}$ & $2.73(0.67)$ & $\begin{array}{l}2.73 \\
(0.83)\end{array}$ & $\begin{array}{l}2.24 \\
(0.80)\end{array}$ & $\begin{array}{l}2.94 \\
(0.47)\end{array}$ & $\begin{array}{l}2.08 \\
(0.83)\end{array}$ \\
\hline \multicolumn{7}{|c|}{ Lymphedema stages } \\
\hline Stage $0 \sim 1$ & $\begin{array}{l}2.16 \\
(0.68)\end{array}$ & $2.30(0.89)$ & $\begin{array}{l}2.12 \\
(0.80) *\end{array}$ & $\begin{array}{l}1.74 \\
(0.81)^{\star}\end{array}$ & $\begin{array}{l}2.55 \\
(0.80)\end{array}$ & $\begin{array}{l}2.12 \\
(0.95)\end{array}$ \\
\hline Stage 2 3 & $\begin{array}{l}2.34 \\
(0.67)\end{array}$ & $2.33(0.82)$ & $\begin{array}{l}2.45 \\
(0.85)\end{array}$ & $\begin{array}{l}2.08 \\
(0.87)\end{array}$ & $\begin{array}{l}2.63 \\
(0.78)\end{array}$ & $\begin{array}{l}2.23 \\
(0.79)\end{array}$ \\
\hline \multicolumn{7}{|c|}{ Number of comorbidities } \\
\hline 0 & $\begin{array}{l}2.21 \\
(0.67)\end{array}$ & $\begin{array}{l}2.27 \\
(0.85) \star \star\end{array}$ & $\begin{array}{l}2.17 \\
(0.78)\end{array}$ & $\begin{array}{l}1.82 \\
(0.80)\end{array}$ & $\begin{array}{l}2.59 \\
(0.77)\end{array}$ & $\begin{array}{l}2.20 \\
(0.94)\end{array}$ \\
\hline 1 & $\begin{array}{l}2.12 \\
(0.67)\end{array}$ & $2.11(0.77)$ & $\begin{array}{l}2.21 \\
(0.83)\end{array}$ & $\begin{array}{l}1.79 \\
(0.85)\end{array}$ & $\begin{array}{l}2.44 \\
(0.79)\end{array}$ & $\begin{array}{l}2.03 \\
(0.72)\end{array}$ \\
\hline 2 & $\begin{array}{l}2.50 \\
(0.79)\end{array}$ & $2.80(0.75)$ & $\begin{array}{l}2.35 \\
(0.86)\end{array}$ & $\begin{array}{l}2.20 \\
(0.93)\end{array}$ & $\begin{array}{l}2.73 \\
(0.94)\end{array}$ & $\begin{array}{l}2.46 \\
(1.05)\end{array}$ \\
\hline$\geq 3$ & $\begin{array}{l}2.51 \\
(0.71)\end{array}$ & $2.97(1.20)$ & $\begin{array}{l}2.76 \\
(0.94)\end{array}$ & $\begin{array}{l}2.12 \\
(1.13)\end{array}$ & $\begin{array}{l}2.98 \\
(0.69)\end{array}$ & $\begin{array}{l}1.79 \\
(0.44)\end{array}$ \\
\hline
\end{tabular}




\begin{tabular}{|c|c|c|c|c|c|c|}
\hline Variables & $\begin{array}{l}\text { Total } \\
\text { QOL }\end{array}$ & $\begin{array}{l}\text { Physical } \\
\text { Impairments }\end{array}$ & $\begin{array}{l}\text { Daily } \\
\text { Life }\end{array}$ & $\begin{array}{l}\text { Social } \\
\text { Life }\end{array}$ & $\begin{array}{l}\text { Mental } \\
\text { Condition }\end{array}$ & $\begin{array}{l}\text { Therapy } \\
\text { of } \\
\text { Lymphatic } \\
\text { Disorder }\end{array}$ \\
\hline \multicolumn{7}{|c|}{ Time since diagnosis } \\
\hline a<6 (months) & $\begin{array}{l}2.36 \\
(0.70)\end{array}$ & $2.54(0.90)$ & $\begin{array}{l}2.31 \\
(0.73)\end{array}$ & $\begin{array}{l}1.99 \\
(0.85)\end{array}$ & $\begin{array}{l}2.72 \\
(0.79)\end{array}$ & $\begin{array}{l}2.27 \\
(0.92)\end{array}$ \\
\hline $6 \leq \mathrm{a}<12$ (months) & $\begin{array}{l}2.19 \\
(0.62)\end{array}$ & $2.20(0.89)$ & $\begin{array}{l}2.22 \\
(0.92)\end{array}$ & $\begin{array}{l}1.65 \\
(0.80)\end{array}$ & $\begin{array}{l}2.65 \\
(0.79)\end{array}$ & $\begin{array}{l}2.23 \\
(0.84)\end{array}$ \\
\hline $1 \leq a<2$ (years) & $\begin{array}{l}2.15 \\
(0.63)\end{array}$ & $2.23(0.80)$ & $\begin{array}{l}2.16 \\
(0.73)\end{array}$ & $\begin{array}{l}1.89 \\
(0.92)\end{array}$ & $\begin{array}{l}2.50 \\
(0.90)\end{array}$ & $\begin{array}{l}1.98 \\
(0.76)\end{array}$ \\
\hline $2 \leq a<5$ (years) & $\begin{array}{l}2.13 \\
(0.67)\end{array}$ & $2.19(0.81)$ & $\begin{array}{l}2.11 \\
(0.79)\end{array}$ & $\begin{array}{l}1.78 \\
(0.82)\end{array}$ & $\begin{array}{l}2.47 \\
(0.71)\end{array}$ & $\begin{array}{l}2.10 \\
(0.95)\end{array}$ \\
\hline $5 \leq a<10$ (years) & $\begin{array}{l}2.38 \\
(0.79)\end{array}$ & $2.49(0.89)$ & $\begin{array}{l}2.50 \\
(1.09)\end{array}$ & $\begin{array}{l}1.96 \\
(0.81)\end{array}$ & $\begin{array}{l}2.63 \\
(0.89)\end{array}$ & $\begin{array}{l}2.34 \\
(1.01)\end{array}$ \\
\hline$a \geq 10$ (years) & $\begin{array}{l}2.20 \\
(0.81)\end{array}$ & $2.07(1.00)$ & $\begin{array}{l}2.29 \\
(1.89)\end{array}$ & $\begin{array}{l}1.77 \\
(0.90)\end{array}$ & $\begin{array}{l}2.56 \\
(0.82)\end{array}$ & $\begin{array}{l}2.27 \\
(0.82)\end{array}$ \\
\hline \multicolumn{7}{|c|}{ Number of symptoms } \\
\hline 0 & $\begin{array}{l}1.56 \\
(0.42) \star \star\end{array}$ & $1.40(0.50) \star$ & $\begin{array}{l}1.44 \\
(0.25)^{\star \star}\end{array}$ & $\begin{array}{l}1.44 \\
(0.69) \star \star\end{array}$ & $\begin{array}{l}1.80 \\
(0.55)^{\star \star}\end{array}$ & $\begin{array}{l}1.67 \\
(1.06) \star \star\end{array}$ \\
\hline 1 & $\begin{array}{l}2.07 \\
(0.62)\end{array}$ & $2.17(0.85)$ & $\begin{array}{l}2.07 \\
(0.70)\end{array}$ & $\begin{array}{l}1.68 \\
(0.71)\end{array}$ & $\begin{array}{l}2.49 \\
(0.81)\end{array}$ & $\begin{array}{l}1.98 \\
(0.85)\end{array}$ \\
\hline 2 & $\begin{array}{l}2.06 \\
(0.64)\end{array}$ & $2.13(0.89)$ & $\begin{array}{l}2.08 \\
(0.79)\end{array}$ & $\begin{array}{l}1.60 \\
(0.74)\end{array}$ & $\begin{array}{l}2.50 \\
(0.81)\end{array}$ & $\begin{array}{l}1.99 \\
(0.82)\end{array}$ \\
\hline$\geq 3$ & $\begin{array}{l}2.47 \\
(0.66)\end{array}$ & $2.57(0.78)$ & $\begin{array}{l}2.46 \\
(0.82)\end{array}$ & $\begin{array}{l}2.13 \\
(0.89)\end{array}$ & $\begin{array}{l}2.75 \\
(0.74)\end{array}$ & $\begin{array}{l}2.39 \\
(0.86)\end{array}$ \\
\hline \multicolumn{7}{|l|}{ Degree of pain } \\
\hline No pain & $\begin{array}{l}2.06 \\
(0.63) \star \star\end{array}$ & $\begin{array}{l}2.09 \\
(0.81)^{\star \star \star}\end{array}$ & $\begin{array}{l}2.10 \\
(0.75)^{\star \star}\end{array}$ & $\begin{array}{l}1.68 \\
(0.72)^{\star \star}\end{array}$ & $\begin{array}{l}2.44 \\
(0.76)^{\star \star}\end{array}$ & $\begin{array}{l}2.00 \\
(0.87) \star \star\end{array}$ \\
\hline Mild pain & $\begin{array}{l}2.31 \\
(0.68)\end{array}$ & $2.36(0.73)$ & $\begin{array}{l}2.21 \\
(0.78)\end{array}$ & $\begin{array}{l}2.03 \\
(0.94)\end{array}$ & $\begin{array}{l}2.63 \\
(0.83)\end{array}$ & $\begin{array}{l}2.31 \\
(0.87)\end{array}$ \\
\hline Moderate pain & $\begin{array}{l}2.85 \\
(0.56)\end{array}$ & $3.27(0.58)$ & $\begin{array}{l}2.82 \\
(0.87)\end{array}$ & $\begin{array}{l}2.40 \\
(0.92)\end{array}$ & $\begin{array}{l}3.12 \\
(0.64)\end{array}$ & $\begin{array}{l}2.63 \\
(0.85)\end{array}$ \\
\hline \multicolumn{7}{|c|}{$\begin{array}{l}\text { Sexual distress caused by } \\
\text { Lymphedema }\end{array}$} \\
\hline None & $\begin{array}{l}2.14 \\
(0.67) \star \star\end{array}$ & $2.24(0.87)^{\star}$ & $\begin{array}{l}2.16 \\
(0.80) \star\end{array}$ & $\begin{array}{l}1.73 \\
(0.76) \star \star\end{array}$ & $\begin{array}{l}2.49 \\
(0.76) \star \star\end{array}$ & $\begin{array}{l}2.12 \\
(0.91)\end{array}$ \\
\hline Yes & $\begin{array}{l}2.62 \\
(0.63)\end{array}$ & $2.65(0.76)$ & $\begin{array}{l}2.56 \\
(0.78)\end{array}$ & $\begin{array}{l}2.54 \\
(0.91)\end{array}$ & $\begin{array}{l}3.00 \\
(0.82)\end{array}$ & $\begin{array}{l}2.37 \\
(0.78)\end{array}$ \\
\hline${ }^{* \star} p<0.01 ;{ }^{*} p<0.05$ & & & & & & \\
\hline
\end{tabular}




\subsection{Correlations between acceptance of disability, coping style, perceived social support and quality of life}

The results of Pearson correlation analysis indicated that total QOL, physical impairments, and mental condition were negatively associated with total acceptance, enlargement, subordination, containment, transformation, total perceived social support, family support, friend support, and positively related to avoidance and acceptance-resignation. Additionally, the results also showed that daily life, social life, and therapy of lymphatic disorder have significantly negative relations to the total acceptance, enlargement, subordination, containment, transformation, total perceived social support, family support, friend support, and significantly positive associations with acceptance-resignation. (Table 4)

Table 4

The correlation between acceptance of disability, coping style, and perceived social support, and quality of life

\begin{tabular}{|c|c|c|c|c|c|c|}
\hline Dimensions & $\begin{array}{l}\text { Total } \\
\text { QOL }\end{array}$ & $\begin{array}{l}\text { Physical } \\
\text { impairments }\end{array}$ & $\begin{array}{l}\text { Daily } \\
\text { life }\end{array}$ & $\begin{array}{l}\text { Social } \\
\text { life }\end{array}$ & $\begin{array}{l}\text { Mental } \\
\text { condition }\end{array}$ & $\begin{array}{l}\text { Therapy of } \\
\text { lymphatic disorder }\end{array}$ \\
\hline Total acceptance & $-0.636 * *$ & $-0.371 * *$ & $-0.542^{\star *}$ & $-0.546 * \star$ & $-0.658 * *$ & $-0.458 * *$ \\
\hline Enlargement & $-0.323^{* *}$ & $-0.176^{\star}$ & $-0.273^{\star *}$ & $-0.259 * \star$ & $-0.340 * \star$ & $-0.256^{\star \star}$ \\
\hline Subordination & $-0.297 * \star$ & $-0.114^{\star}$ & $-0.246 * \star$ & $-0.249 * *$ & $-0.324 * \star$ & $-0.261^{\star *}$ \\
\hline Containment & -0.621 ** & $-0.384^{\star \star}$ & $-0.555^{\star \star}$ & -0.538 ** & $-0.622^{\star \star}$ & -0.420 ** \\
\hline Transformation & $-0.567 * \star$ & $-0.350 * \star$ & $-0.456 \star \star$ & $-0.502^{\star \star}$ & $-0.599 * *$ & $-0.392 * \star$ \\
\hline Confrontation & -0.073 & -0.052 & -0.046 & -0.092 & -0.070 & -0.039 \\
\hline Avoidance & $0.170 *$ & $0.157 *$ & 0.086 & 0.152 & $0.161 *$ & 0.140 \\
\hline $\begin{array}{l}\text { Acceptance- } \\
\text { resignation }\end{array}$ & $0.546 * \star$ & $0.296 * *$ & $0.496 * *$ & $0.490 * *$ & $0.571 * \star$ & $0.356 * *$ \\
\hline $\begin{array}{l}\text { Total perceived social } \\
\text { support }\end{array}$ & $-0.452^{\star \star}$ & $-0.283^{* *}$ & $-0.369 * \star$ & $-0.404^{\star \star}$ & $-0.457 * \star$ & $-0.319 * *$ \\
\hline Family support & $0.511^{\star \star}$ & $-0.359 * *$ & $-0.452 * *$ & $-0.449 \star \star$ & $-0.444^{\star \star}$ & -0.371 ** \\
\hline Friend support & $0.432^{\star \star}$ & $-0.344 * \star$ & $-0.335^{\star \star}$ & $-0.369 * *$ & $-0.452 * \star$ & $-0.266 * *$ \\
\hline $\begin{array}{l}\text { Significant other } \\
\text { support }\end{array}$ & -0.116 & 0.051 & -0.087 & -0.132 & $-0.163^{*}$ & -0.120 \\
\hline${ }^{\star \star} p<0.01 ;{ }^{*} p<0.05$ & & & & & & \\
\hline
\end{tabular}

\subsection{Factors influencing the quality of life in Chinese patients with chronic lymphedema}

Table 5 shows the results of the multivariable linear regression model for quality of life and its five domains. In the total QOL model, total acceptance, number of symptoms, acceptance-resignation, total perceived social support, degree of pain, educational level, and avoidance were significant variables explaining $57.6 \%$ of the variance in QOL ( $F=30.054, P<0.001)$. Additionally, concerning the domains of QOL, three variables (degree of pain, total acceptance, and number of symptoms) accounted for $27.9 \%$ of the variance in physical impairments $(F=20.523, P<0.001)$, four 
variables (total acceptance, number of symptoms, acceptance-resignation, and total perceived social support) accounted for $39.1 \%$ of the variance in daily life $(F=25.341, P<0.001)$, six variables (total acceptance, sexual distress caused by lymphedema, degree of pain, acceptance-resignation, educational level, and total perceived social support) accounted for $47.9 \%$ of the variance in social life $(F=17.719, P<0.001)$, five variables (total acceptance, acceptanceresignation, total perceived social support, number of symptoms, and avoidance) accounted for $53.8 \%$ of the variance in mental condition ( $F=36.560, P<0.001$ ), and three variables (total acceptance, place of residence, and number of symptoms) accounted for $26.6 \%$ of the variance in the therapy of lymphatic disorder $(F=20.561, P<0.001)$. 
Table 5

Multiple linear regression of HRQOL among chronic lymphedema patients $(\mathrm{N}=163)$

\begin{tabular}{|c|c|c|c|c|c|}
\hline Outcome variables & Explanatory variables & $\begin{array}{l}\text { Adjusted } \\
\mathrm{R}^{2}\end{array}$ & $\beta$ & $t$ & $P$ \\
\hline \multirow[t]{7}{*}{ Total QOL } & Total acceptance & 0.576 & -0.274 & -3.621 & $0.000 * \star$ \\
\hline & Number of symptoms & & 0.174 & 3.053 & $0.003 * *$ \\
\hline & Acceptance-resignation & & 0.272 & 3.978 & $0.000 * \star$ \\
\hline & Total perceived social support & & -0.187 & -3.184 & $0.002^{\star \star}$ \\
\hline & Degree of pain & & 0.162 & 2.752 & $0.007 \star \star$ \\
\hline & Educational level & & -0.135 & -2.509 & $0.013^{\star}$ \\
\hline & Avoidance & & 0.125 & 2.370 & $0.019 *$ \\
\hline \multirow[t]{3}{*}{ Physical Impairments } & Degree of pain & 0.279 & 0.308 & 4.124 & $0.000 * *$ \\
\hline & Total acceptance & & -0.262 & -3.734 & $0.000 * \star$ \\
\hline & Number of symptoms & & 0.150 & 2.049 & $0.042^{\star}$ \\
\hline \multirow[t]{4}{*}{ Daily Life } & Total acceptance & 0.391 & -0.262 & -3.047 & $0.003^{\star \star}$ \\
\hline & Number of symptoms & & 0.222 & 3.522 & $0.001^{\star *}$ \\
\hline & Acceptance-resignation & & 0.261 & 3.259 & $0.001^{\star *}$ \\
\hline & Total perceived social support & & -0.145 & -2.083 & $0.039 *$ \\
\hline \multirow[t]{6}{*}{ Social Life } & Total acceptance & 0.479 & -0.190 & -2.254 & $0.026^{*}$ \\
\hline & $\begin{array}{l}\text { Sexual distress caused by } \\
\text { Lymphedema }\end{array}$ & & 0.235 & 3.610 & $0.000 * \star$ \\
\hline & Degree of pain & & 0.197 & 3.241 & $0.001 * \star$ \\
\hline & Acceptance-resignation & & 0.239 & 3.105 & $0.002^{\star \star}$ \\
\hline & Educational level & & -0.149 & -2.484 & $0.014^{\star}$ \\
\hline & Total perceived social support & & -0.158 & -2.409 & $0.017 *$ \\
\hline \multirow[t]{5}{*}{ Mental Condition } & Total acceptance & 0.538 & -0.386 & -5.138 & $0.000 * *$ \\
\hline & Acceptance-resignation & & 0.251 & 3.593 & $0.000 * \star$ \\
\hline & Total perceived social support & & -0.183 & -3.008 & $0.003^{\star \star}$ \\
\hline & Number of symptoms & & 0.142 & 2.567 & $0.011^{*}$ \\
\hline & Avoidance & & 0.125 & 2.308 & $0.022 *$ \\
\hline \multirow{3}{*}{$\begin{array}{l}\text { Therapy of Lymphatic } \\
\text { Disorder }\end{array}$} & Total acceptance & 0.266 & -0.383 & -5.468 & $<0.001 * *$ \\
\hline & Place of residence & & 0.209 & 3.020 & $0.003^{\star \star}$ \\
\hline & Number of symptoms & & 0.203 & 2.927 & $0.004 * \star$ \\
\hline
\end{tabular}




\section{Discussion}

To our knowledge, this is the first study to explore the correlation between acceptance of disability, coping style, perceived social support and quality of life among Chinese patients with chronic lymphedema. Three important findings are drawn from our research. First of all, Chinese patients with chronic lymphedema had moderate levels of QOL, AOD, and PSS and tended to adopt a confrontation coping style. Next, QOL was negatively associated with AOD and PSS, while positively related to avoidance and acceptance-resignation coping styles. At last, the predictors of QOL and its domains included AOD, the number of symptoms, acceptance-resignation, avoidance, the degree of pain, PSS, the educational level, the place of residence, and the sexual distress caused by lymphedema. We deem that our findings will add knowledge to the improvement of QOL in patients with chronic lymphedema.

In our study, patients with chronic lymphedema had moderate levels of QOL. Compared with the results of the study using FLQA-LS by Herberger et al. (2017) [24], the QOL of patients with chronic lymphedema in the Chinese population ( $2.23 \pm 0.68)$ is better than that of the German population $(2.4 \pm 0.8)$; Concerning the different domains of QOL, our study showed that patients with chronic lymphedema had the worst QOL in mental condition (2.58 \pm 0.79$)$, which is in line with the result of Herberger et al. (2.70 \pm 0.90$)$, indicating that chronic lymphedema severely compromised patients' mental health. A possible explanation might be that patients with chronic lymphedema often suffered from decreased self-esteem and self-confidence, depression, frustration, and loss of body image due to changes in their normal functional status and appearance caused by lymphedema [25]. Hence, healthcare staff should pay more attention to the mental well-being of patients and develop intervention programs to help patients cope with psychological issues, thereby improving QOL.

Our study showed that patients with chronic lymphedema had moderate levels of AOD, which was positively correlated to QOL and its specific dimensions, indicating that the higher the levels of AOD, the worse the QOL of patients. Patients with better AOD had good QOL since AOD plays a vital role in helping individuals adjust their value system after experiencing stressful life events, thereby relieving negative perceptions and improving QOL [26]. Among the domains of AOD, the highest score appeared in the "enlargement of scope of values" domain, indicating that even though patients suffered from lymphedema, they were still able to recognize the significance of values, not those assumed to be lost. Given the above finding, AOD can be regarded as an essential factor in the development of intervention programs to improve the QOL of patients with chronic lymphedema.

In our study, we demonstrated that the number of symptoms was associated with QOL of chronic lymphedema patients. Chronic lymphedema patients with more symptoms were likely to have worse QOL. Symptoms associated with chronic lymphedema mainly included swelling, pain, fatigue, heaviness, tightness, numbness, stiffness, and impaired limb mobility, which pose a threat to the patient's psychological health and social functions [27]. Dominick et al.[28] found that there was a positive correlation between the number of symptoms and symptom distress in patients with chronic lymphedema. With the progression of lymphedema, the number of symptoms and the symptom distress experienced by patients increase, which affects their QOL. Therefore, healthcare providers should develop intervention programs for symptoms caused by lymphedema to alleviate the patient's symptom distress and thus improve their QOL.

Our study illustrated that patients with chronic lymphedema are prone to choose confrontation coping style, indicating that the patients tend to treat chronic lymphedema with a positive attitude. The results of our work also suggested that there was a positive relationship between acceptance-resignation and avoidance and QOL, which meant acceptance-resignation or avoidance coping strategies can lead to poor QOL. It was acknowledged that acceptance-resignation and avoidance were considered negative responses to stressful events and had been shown

Page $16 / 21$ 
to elicit a negative impact on QOL [29]. In the short term, patients may be able to relieve stress by acceptanceresignation or avoidance coping, however, excessive use of negative coping strategies can result in severe psychological problems, aggravate patients' health outcomes, and thus impairs patients' QOL [30]. Thus, based on our findings, interventions targeted at negative coping styles might have a better effect on the ameliorations of QOL for patients with chronic lymphedema.

In the current study, patients with chronic lymphedema had moderate levels of PSS, which was negatively associated with QOL. This implies that patients with chronic lymphedema who perceived more social support may have a better QOL. It is widely known that we humans are "social beings" with complicated social networks that can influence various aspects of an individual's health status [31]. Social support can produce helpful effects directly or act as a buffer to protect individuals from adverse effects when facing stress. Moreover, some hypotheses suggested that social support might indirectly exert a positive effect on patient's immune systems, thus accelerate their recovery from disease and improve their QOL [32]. Among all the domains, family support and friend support are significantly related to QOL, indicating that family and friend support may be crucial for patients with chronic lymphedema. Given these findings, it is advocated that PSS should be recognized and promoted by medical staff when taking care of chronic lymphedema patients.

The finding of our study showed that the degree of pain was positively correlated with patients' QOL, indicating that patients with higher degrees of pain were likely to have worse QOL. Additionally, in or study, measured by VAS, $61.3 \%$ of Chinese patients with chronic lymphedema had no pain, $25.2 \%$ had mild pain, and $13.5 \%$ had moderate pain, while the study conducted by Herberger et al. [24] in the German population showed that $45 \%$ of patients had no pain, $16 \%$ had mild pain, $24 \%$ had moderate pain, and $14 \%$ had severe pain. This means that chronic lymphedema patients in China suffer less pain than those in Germany. Given this finding, healthcare professionals should assess the patient's pain promptly and provide professional guidance to alleviate their pain, thereby improving their QOL.

In our study, patients with low educational levels are closely related to poor QOL. Education may have an impact on QOL through cognition [33]. Patients with different educational backgrounds had different responses to diseases and treatments. Compared with patients with low levels of education, high-educated patients may be more adaptable to the changes brought about by the disease because they have a deeper understanding of the information provided by healthcare providers [34]. Hence, the finding of our study may imply that chronic lymphedema patients with low educational levels required more interventions aimed at improving their QOL.

Sexual distress refers to the different negative emotional reactions that people experience in terms of their sexuality, such as frustration, bother, anxiety, which ultimately have a negative impact on QOL [35]. In our study, we demonstrate that sexual distress is positively associated with QOL, which is mainly manifested in the social life domain. This means that chronic lymphedema patients with sexual distress caused by lymphedema have poor QOL. Therefore, healthcare staff should promptly evaluate patients' sexual distress. For patients with sexual distress, healthcare staff should adopt targeted intervention programs to help patients solve problems in their sexual life, reduce their sexual distress, and thus improve their QOL.

It has been documented that residing in the rural area has adverse effects on QOL [36]. Our study confirmed that patients with chronic lymphedema who were residing in a rural area had worse QOL compared to those who were from urban areas, which mainly manifested in the therapy of lymphatic disorder domain. This might be due to the fact that residing in rural areas is commonly related to delays in diagnosis, the challenges of long-distance travel to urban medical centers, and limited access to lymphedema services [37], which place a burden on chronic lymphedema patients. Hence, given the lack of lymphedema-related health resources in rural areas, developing

Page $17 / 21$ 
telemedicine-based and navigator-based outreach programs that can bring lymphedema care nearer to rural areas may increase rural patients' QOL.

There are several limitations to this study. To begin with, this study was conducted in five tertiary hospitals in Guangdong province, China, which might not truly represent all chronic lymphedema patients in China, thus limiting the nationwide generalization of the findings. Moreover, the findings were not able to provide evidence for causation because of the cross-sectional study design. Additionally, there might be a degree of social desirability bias due to the use of self-report questionnaires.

\section{Conclusion}

This study adds to the limited research on the QOL of Chinese patients with chronic lymphedema. It appears that chronic lymphedema patients in China have moderate levels of QOL, though many of them still have difficulties with physical impairments, daily life, social life, mental health, and treatment. We highlight the importance of AOD, coping style, and PSS in ensuring high levels of QOL among patients with chronic lymphedema. Moreover, there were many influencing factors significantly associated with QOL related to specific domains, including AOD, number of symptoms, acceptance-resignation, avoidance, pain, PSS, educational level, place of residence, and sexual distress caused by lymphedema. Healthcare staff ought to develop targeted interventions based on these factors to improve the QOL of chronic lymphedema patients.

\section{Declarations}

\section{Funding}

This research received no specific grant from any funding agency in the public, commercial, or not-for-profit sectors.

\section{Conflicts of interest}

The authors declare that they have no conflict of interest.

\section{Availability of data and material}

The data are available from the corresponding author on reasonable request.

\section{Code availability}

Not applicable.

\section{Author contributors}

ZH contributed to the study's conception and design. Data collection and analysis were performed by ZH and SW. The first draft of the manuscript was written by $\mathrm{ZH}$. SW reviewed and edited the manuscript. Both authors read and approved the final manuscript.

\section{Ethical approval}

All procedures performed in studies involving human participants were following the ethical standards of the institutional and/or national research committee (Medical Ethics Committee of * Affiliated Hospital of * University [2019] 02-545-01) and with the 1964 Helsinki declaration and its later amendments or comparable ethical standards. 


\section{Consent to participate}

All patients agreed to participate in this study.

\section{Consent for publication}

All participants agreed to publish their data.

\section{References}

1. Mercier G, Pastor J, Moffatt C, Franks P, Quéré I (2019) LIMPRINT: Health-Related Quality of Life in Adult Patients with Chronic Edema Lymphatic research and biology 17: 163-167. https://doi.org/10.1089//rb.2018.0084

2. Lopez M, Roberson ML, Strassle PD, Ogunleye A (2020) Epidemiology of Lymphedema-related admissions in the United States: 2012-2017 Surgical oncology 35: 249-253. https://doi.org/10.1016/j.suronc.2020.09.005

3. Ridner SH, Doersam JK, Stolldorf DP, Dietrich MS (2018) Development and Validation of the Lymphedema Symptom Intensity and Distress Survey-Lower Limb Lymphatic research and biology 16: 538-546. https://doi.org/10.1089//rb.2017.0069

4. Gutknecht M, Herberger K, Klose K, Purwins S, Dietz D, Blome C, Augustin M (2017) Cost-of-illness of patients with lymphoedema Journal of the European Academy of Dermatology and Venereology : JEADV 31: 1930-1935. https://doi.org/10.1111/jdv.14442

5. Tzani I, Tsichlaki M, Zerva E, Papathanasiou G, Dimakakos E (2018) Physiotherapeutic rehabilitation of lymphedema: state-of-the-art Lymphology 51

6. Borman P, Moffatt C, Murray S, Yaman A, Denizli M, Dalyan M, Unsal-Delialioğlu S, Eyigör S, Ayhan F, Çakıt BD, Vural S, Özdemir O, Kurt E, Çelik EC, Cerrahoğlu L, Kepekçi M, Terzioğlu F, Donmez AA (2019) LIMPRINT Study: The Turkish Experience Lymphatic research and biology 17: 202-210. https://doi.org/10.1089//rb.2019.0015

7. Zhang Q, Xiao S, Yan L, Sun L, Wang Y, Huang M (2019) Psychosocial Predictors of Adjustment to Disability Among Patients With Breast Cancer: A Cross-Sectional Descriptive Study The journal of nursing research : JNR 27: e15. https://doi.org/10.1097/jnr.0000000000000283

8. Wright BA (1983) Physical disability - a psychosocial approach, 2nd ed. HarperCollins Publishers, New York, NY, US

9. Yehene E, Lichtenstern G, Harel Y, Druckman E, Sacher Y (2019) Self-efficacy and acceptance of disability following mild traumatic brain injury: A pilot study Applied neuropsychology Adult: 1-10.

https://doi.org/10.1080/23279095.2019.1569523

10. Zhang X, Liu Y, Deng X, Deng C, Pan Y, Hu A (2019) The Correlation Between Quality of Life and Acceptability of Disability in Patients With Facial Burn Scars Front Bioeng Biotechnol 7: 329.

https://doi.org/10.3389/fbioe.2019.00329

11. Folkman S, Lazarus RS, Gruen RJ, DeLongis A (1986) Appraisal, coping, health status, and psychological symptoms J Pers Soc Psychol 50: 571-579.

12. Torkzadeh F, Danesh M, Mirbagher L, Daghaghzadeh H, Emami MH (2019) Relations between Coping Skills, Symptom Severity, Psychological Symptoms, and Quality of Life in Patients with Irritable Bowel Syndrome International journal of preventive medicine 10: 72. https://doi.org/10.4103/ijpvm.IJPVM_464_17

13. Teo I, Fingeret MC, Liu J, Chang DW (2016) Coping and quality of life of patients following microsurgical treatment for breast cancer-related lymphedema Journal of health psychology 21: 2983-2993. https://doi.org/10.1177/1359105315589801

Page 19/21 
14. Lo Buono V, Corallo F, Bramanti P, Marino S (2017) Coping strategies and health-related quality of life after stroke Journal of health psychology 22: 16-28. https://doi.org/10.1177/1359105315595117

15. Howard S, Creaven A-M, Hughes BM, O'Leary ÉD, James JE (2017) Perceived social support predicts lower cardiovascular reactivity to stress in older adults Biol Psychol 125: 70-75.

https://doi.org/10.1016/j.biopsycho.2017.02.006

16. Wang J, Mann F, Lloyd-Evans B, Ma R, Johnson S (2018) Associations between loneliness and perceived social support and outcomes of mental health problems: a systematic review BMC psychiatry 18: 156. https://doi.org/10.1186/s12888-018-1736-5

17. Tsai W, Lu Q (2018) Perceived Social Support Mediates the Longitudinal Relations between Ambivalence over Emotional Expression and Quality of Life among Chinese American Breast Cancer Survivors International journal of behavioral medicine 25: 368-373. https://doi.org/10.1007/s12529-017-9705-9

18. Hirani SS, Norris CM, Van Vliet KJ, Van Zanten SV, Karmaliani R, Lasiuk G (2018) Social support intervention to promote resilience and quality of life in women living in Karachi, Pakistan: a randomized controlled trial Int $\mathrm{J}$ Public Health 63: 693-702. https://doi.org/10.1007/s00038-018-1101-y

19. Livneh H, F. Antonak R (1997) Psychosocial Adaptation to Chronic Illness and Disability.

20. Chen NI, Yun C, Xiao-chun SUN, Jing-jing HU (2009) Relationship between acceptance of disability and posttraumatic stress response in patients with brachial plexus injury Chinese Journal of Nursing 44: 677-680.

21. Shen XH, \& Jiang, Q. J (2000) Report on application of Chinese version of MCMQ among 701 patients Chinese Journal of Behavioral Medical Science 9: 18-20.

22. Huang L, Jiang QJ, Ren WH (1996) The correlational study on coping modes, social support and psychosomatic symptoms among cancer patients Chinese Mental Health Journal 8: 199-201.

23. Augustin M, Conde Montero E, Hagenstrom K, Herberger K, Blome C (2018) Validation of a short-form of the Freiburg Life Quality Assessment for lymphoedema (FLQA-LS) instrument The British journal of dermatology 179: 1329-1333. https://doi.org/10.1111/bjd.16902

24. Herberger K, Blome C, Heyer K, Ellis F, Munter KC, Augustin M (2017) Quality of life in patients with primary and secondary lymphedema in the community Wound Repair and Regeneration 25: 466-473.

25. Taghian NR, Miller CL, Jammallo LS, O'Toole J, Skolny MN (2014) Lymphedema following breast cancer treatment and impact on quality of life: a review Critical reviews in oncology/hematology 92: 227-234. https://doi.org/10.1016/j.critrevonc.2014.06.004

26. Zhang TL, Hu AL, Xu HL, Zheng MC, Liang MJ (2013) Patients after colostomy: relationship between quality of life and acceptance of disability and social support Chin Med J (Engl) 126: 4124-4131.

27. Shi S, Lu Q, Fu MR, Ouyang Q, Liu C, Lv J, Wang Y (2016) Psychometric properties of the Breast Cancer and Lymphedema Symptom Experience Index: The Chinese version European journal of oncology nursing : the official journal of European Oncology Nursing Society 20: 10-16. https://doi.org/10.1016/j.ejon.2015.05.002

28. Dominick SA, Natarajan L, Pierce JP, Madanat H, Madlensky L (2014) The psychosocial impact of lymphedemarelated distress among breast cancer survivors in the WHEL Study Psycho-oncology 23: 1049-1056. https://doi.org/10.1002/pon.3510

29. Du R, Wang P, Ma L, Larcher LM, Wang T, Chen C (2020) Health-related quality of life and associated factors in patients with myocardial infarction after returning to work: a cross-sectional study Health and quality of life outcomes 18: 190. https://doi.org/10.1186/s12955-020-01447-4

30. Liu Z, Zhou X, Zhang W, Zhou L (2020) Resilience and its correlates among first ischemic stroke survivors at acute stage of hospitalization from a tertiary hospital in China囚a cross-sectional study Aging \& mental health 24: 
828-836. https://doi.org/10.1080/13607863.2018.1550630

31. Jalali-Farahani S, Amiri P, Karimi M, Vahedi-Notash G, Amirshekari G, Azizi F (2018) Perceived social support and health-related quality of life (HRQoL) in Tehranian adults: Tehran lipid and glucose study Health and quality of life outcomes 16: 90. https://doi.org/10.1186/s12955-018-0914-y

32. Zimet GD, Powell SS, Farley GK, Werkman S, Berkoff KA (1990) Psychometric characteristics of the Multidimensional Scale of Perceived Social Support Journal of personality assessment 55: 610-617.

33. Xie Y, Yu Y, Wang J-X, Yang X, Zhao F, Ma J-Q, Chen Z-Y, Liang F-R, Zhao L, Cai D-J, Yang C-X (2020) Healthrelated quality of life and its influencing factors in Chinese with knee osteoarthritis Quality of life research : an international journal of quality of life aspects of treatment, care and rehabilitation 29: 2395-2402. https://doi.org/10.1007/s11136-020-02502-9

34. Barbareschi G, Sanderman R, Leegte IL, van Veldhuisen DJ, Jaarsma T (2011) Educational level and the quality of life of heart failure patients: a longitudinal study Journal of cardiac failure 17: 47-53. https://doi.org/10.1016/j.cardfail.2010.08.005

35. Santos-Iglesias P, Mohamed B, Walker LM (2018) A Systematic Review of Sexual Distress Measures The journal of sexual medicine 15: 625-644. https://doi.org/10.1016/j.jsxm.2018.02.020

36. Noonan D, LeBlanc M, Conley C, Benecha H, Leak-Bryant A, Peter K, Zimmerman S, Mayer D, Smith S (2020) Quality of Life and Impact of Cancer: Differences in Rural and Nonrural Non-Hodgkin's Lymphoma Survivors J Rural Health 36: 536-542. https://doi.org/10.1111/jrh.12420

37. Yihedego E, Aga F, Gela D, Boka A (2020) Quality of Life and Associated Factors Among Family Caregivers of Adult Cancer Patients in Addis Ababa, Ethiopia Cancer Manag Res 12: 10047-10054. https://doi.org/10.2147/cmar.S266416 